\title{
The impact of regionalized trauma care on the distribution of severely injured patients in the Netherlands
}

\author{
Suzan Dijkink ${ }^{1}$ - Erik W. van Zwet ${ }^{2} \cdot$ Pieta Krijnen $^{1} \cdot$ Luke P. H. Leenen $^{3} \cdot$ Frank W. Bloemers $^{4}$. \\ Michael J. R. Edwards ${ }^{5}$. Dennis Den Hartog ${ }^{6} \cdot$ Peter A. Leenhouts $^{7} \cdot$ Martijn Poeze $^{8} \cdot$ W. Richard Spanjersberg ${ }^{9}$. \\ Klaus W. Wendt ${ }^{10} \cdot$ Ralph J. De Wit ${ }^{11} \cdot$ Stefan W. A. M. Van Zuthpen ${ }^{12} \cdot$ Inger B. Schipper ${ }^{1}$
}

Received: 13 October 2020 / Accepted: 5 February 2021 / Published online: 12 March 2021

(c) The Author(s) 2021

\begin{abstract}
Background Twenty years ago, an inclusive trauma system was implemented in the Netherlands. The goal of this study was to evaluate the impact of structured trauma care on the concentration of severely injured patients over time.

Methods All severely injured patients (Injury Severity Score [ISS] $\geq 16$ ) documented in the Dutch Trauma Registry (DTR) in the calendar period 2008-2018 were included for analysis. We compared severely injured patients, with and without severe neurotrauma, directly brought to trauma centers (TC) and non-trauma centers (NTC). The proportion of patients being directly transported to a trauma center was determined, as was the total Abbreviated Injury Score (AIS), and ISS.

Results The documented number of severely injured patients increased from 2350 in 2008 to 4694 in 2018. During this period, on average, $70 \%$ of these patients were directly admitted to a TC (range 63-74\%). Patients without severe neurotrauma had a lower chance of being brought to a TC compared to those with severe neurotrauma. Patients directly presented to a TC were more severely injured, reflected by a higher total AIS and ISS, than those directly transported to a NTC.

Conclusion Since the introduction of a well-organized trauma system in the Netherlands, trauma care has become progressively centralized, with more severely injured patients being directly presented to a TC. However, still $30 \%$ of these patients is initially brought to a NTC. Future research should focus on improving pre-hospital triage to facilitate swift transfer of the right patient to the right hospital.
\end{abstract}

Keywords Trauma system $\cdot$ The Netherlands $\cdot$ Regionalization of care $\cdot$ Trauma care

Suzan Dijkink

s.dijkink@lumc.nl

1 Department of Trauma Surgery, Leiden University Medical Center, Post zone K6-R, P.O. Box 9600, 2300 RC Leiden, The Netherlands

2 Department of Medical Statistics and Bioinformatics, Leiden University Medical Center, Leiden, The Netherlands

3 Department of Surgery, University Medical Center Utrecht, Utrecht, The Netherlands

4 Department of Surgery, VU University Medical Center, Amsterdam, The Netherlands

5 Department of Trauma Surgery, Radboud University Medical Center, Nijmegen, The Netherlands

6 Trauma Research Unit, Department of Surgery, Erasmus MC, University Medical Center Rotterdam, Rotterdam, The Netherlands
7 Department of Surgery, Academic Medical Center, Amsterdam, The Netherlands

8 Department of Surgery, Maastricht University Medical Center, Maastricht, The Netherlands

9 Department of Trauma Surgery, Isala Klinieken, Zwolle, The Netherlands

10 Department of Trauma Surgery, University Medical Center Groningen, University of Groningen, Groningen, the Netherlands

11 Department of Trauma Surgery, Medisch Spectrum Twente, Enschede, The Netherlands

12 Department of Surgery, Elisabeth Tweesteden Ziekenhuis, Tilburg, The Netherlands 


\section{Background}

Following concerns about the organization of both prehospital and in-hospital trauma care and the increased public awareness about the importance of well-organized acute care [1, 2], the Dutch government appointed ten trauma centers (TC) in 1998 [3, 4]. Currently, the Dutch trauma care is organized in eleven trauma regions and resembles the American trauma system based on the criteria set by the American College of Surgeons Committee on Trauma [5]. Each region has a catchment area of at least 1.2 million inhabitants with one coordinating TC and several non-trauma centers (NTC) in every region. Since the introduction of the regionalized trauma care, several quality measures were deployed, such as a mandatory participation in the Dutch Trauma Registry [DTR] (2008), which led to $100 \%$ participation by all emergency departments in the registry in 2015. Also, level criteria for trauma- and non-trauma centers (2013), and certification for trauma surgeons were implemented. In 2010, ten years after the introduction of well-organized trauma systems, the overall mortality risk after trauma was found to be reduced by $16 \%$ [6]. In the past decade, further development of trauma care in the Netherlands concerned, amongst others, the regionalization of the ambulance care including an update of the national guideline for emergency medical service providers (2011), 24/7 availability of helicopter emergency services (HEMS) for acute trauma (2011), yearly quality reports by the Dutch Trauma Registry (2012) and the introduction of trauma-related quality indicators by the Dutch government (2015) (Fig. 1).

Twenty years after the introduction of trauma systems in the Netherlands knowledge about parameters that may influence the distribution of trauma patients is relevant, per se and as a prelude to the analysis of the clinical effects of this concentration of care over time. The objective of this study is to describe the impact of structured trauma care on the distribution of severely injured patients between trauma centers (TC) and non-trauma centers (NTC).

\section{Materials and methods}

\section{Patients and data}

Patients admitted to either one of the appointed regional trauma centers (TCs) or to a non-trauma center (NTCs) are registered in the Dutch Trauma Registry (DTR). This retrospective cohort study included all severely injured patients (Injury Severity Score [ISS] $\geq 16$ ) who were registered in the DTR during the calendar period 2008-2018. Up to 2015, injury coding and calculation of the ISS [7] in the DTR was based on the Abbreviated Injury Scale (AIS) version 1998 [8] and after 2015 on the AIS version 2008 [9]. To enable a comparison of patients' injury severity over time, a tool developed by Palmer et al. was used to reclassify all AIS1998 injury codes to AIS2008 injury codes [10].

Since 2008, all eleven coordinating TCs contribute data of their admitted trauma patients to the DTR. During the study period (2008-2018), the participation of NTCs in the DTR increased from 62\% in 2008 to (near) $100 \%$ in 2018 (Table 1). From 2014 on, all Dutch hospitals participate in the DTR.

The distinction between TCs and NTCs is based on the set of trauma center- criteria established by the Dutch Society for Trauma Surgery. TCs (level I centers) in the Netherlands need to have 24/7 multidisciplinary trauma team availability and are equipped for multidisciplinary management of severely injured patients, including the

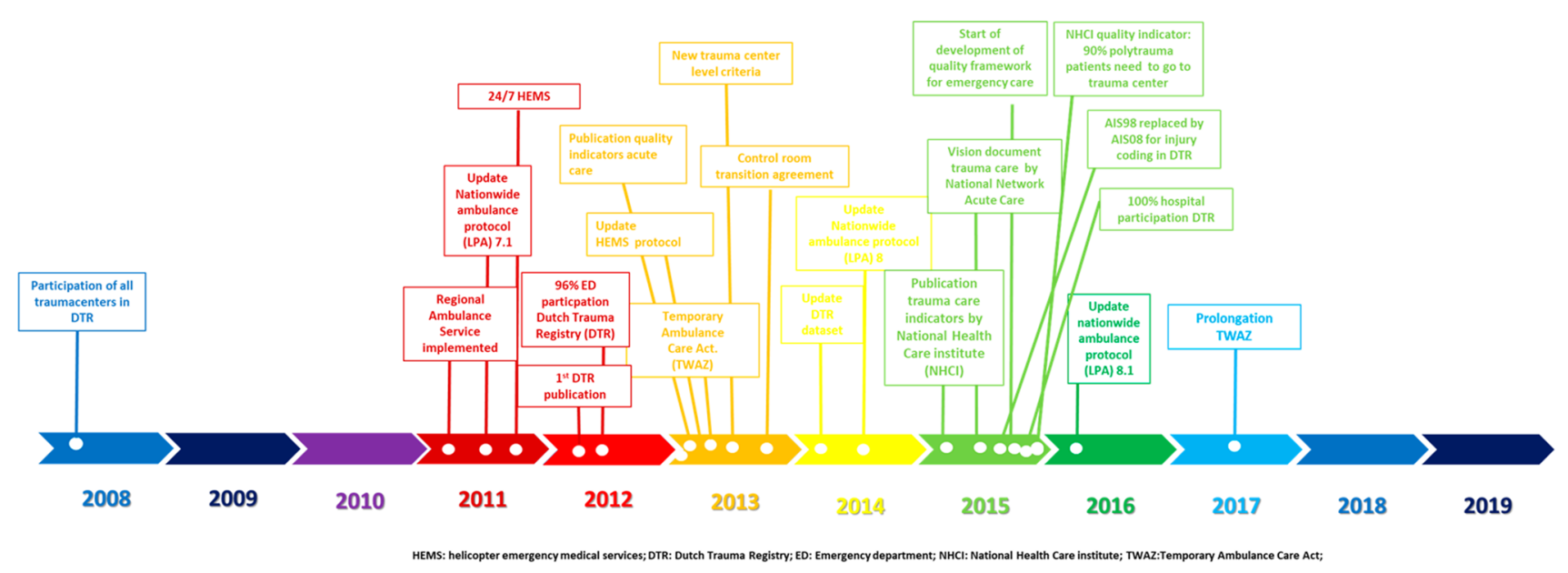

Fig. 1 Changes in organization of trauma care in the Netherlands 


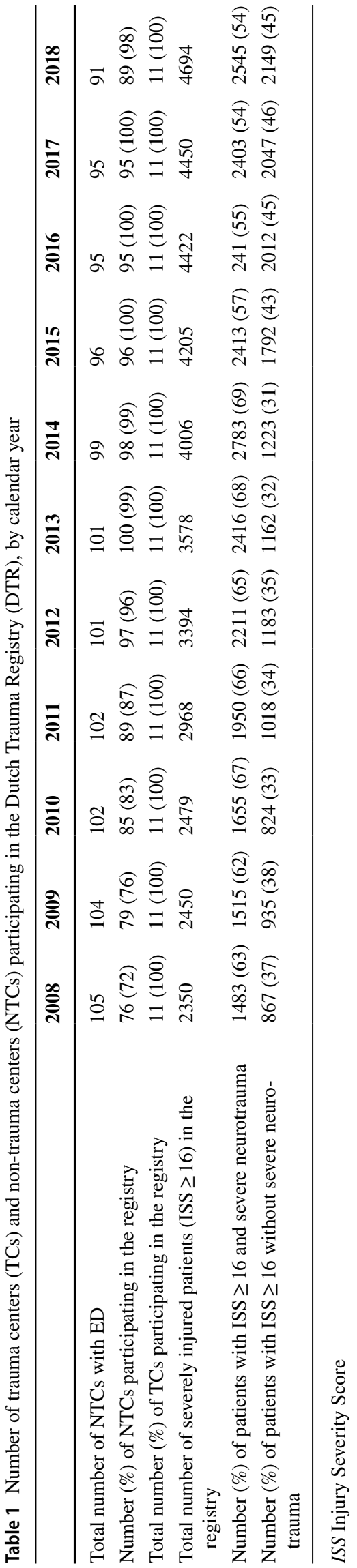

presence of facilities, such as 24/7 angio-interventions, intensive care and specialties like neurosurgery and cardiothoracic surgery. The NTCs are well-equipped traumahospitals but lack the $24 / 7$ presence of multidisciplinary trauma teams, and are not appointed primarily to provide care to severely injured patients.

\section{Statistical analysis}

The statistical analyses were performed in R [11] for three types of patients:

A) all severely injured patients (ISS $\geq 16$ ),

B) patients with ISS $\geq 16$ and severe (head-AIS $\geq 3$ ) neurotrauma, and

C) patients with ISS $\geq 16$ without neurotrauma or with only mild to moderate (AIS $\leq 2$ ) neurotrauma.

Separate analysis of the subgroups with and without severe neurotrauma was considered relevant, since a large part of the patients with ISS $\geq 16$ has severe neurotrauma.

First, the distribution of severely injured patients who were directly brought to a TC and those who were directly brought to a NTC was described over time (Fig. 2).

To assess a potential trend in the proportion of severely injured patients directly brought to a TC over time, the proportion per year was modelled with adjustment for case mix variables (Fig. 3). For this purpose, the PSNL15 case mix model was used, which was developed by the National Network Acute Care (Landelijk Netwerk Acute Zorg, LNAZ), based on the TRISS model [12] and adjusted to the Dutch population [13]. The PSNL15 case mix model includes factors associated with the survival of trauma patients, such as trauma mechanism, vital signs on admission, age and ISS [13]. The proportion of patients that were directly brought to a TC was also corrected for the centers that did not participate in the DTR from the start in 2007. Multiple imputation was used to estimate the number of (severely) injured patients in NTCs for the calendar years in which these centers did not report to the DTR. The multiple imputation for the not reported years was based on the number of and trend in the observed numbers of patients these centers had reported in later years.

Second, the median ISS (Fig. 4) and median total AIS (calculated as the sum of all separate AIS severity codes per patient) (Fig. 5) of patients brought to the TCs and NTCs were described over time. The median total AIS was analyzed as several studies have shown that the low interobserver reliability of the ISS limits its use for benchmarking trauma system performance $[14,15]$. Total AIS might be a useful marker of injury severity because it includes all injuries (i.e. multiple injuries in one body region) [16]. 


\section{A. All patients}

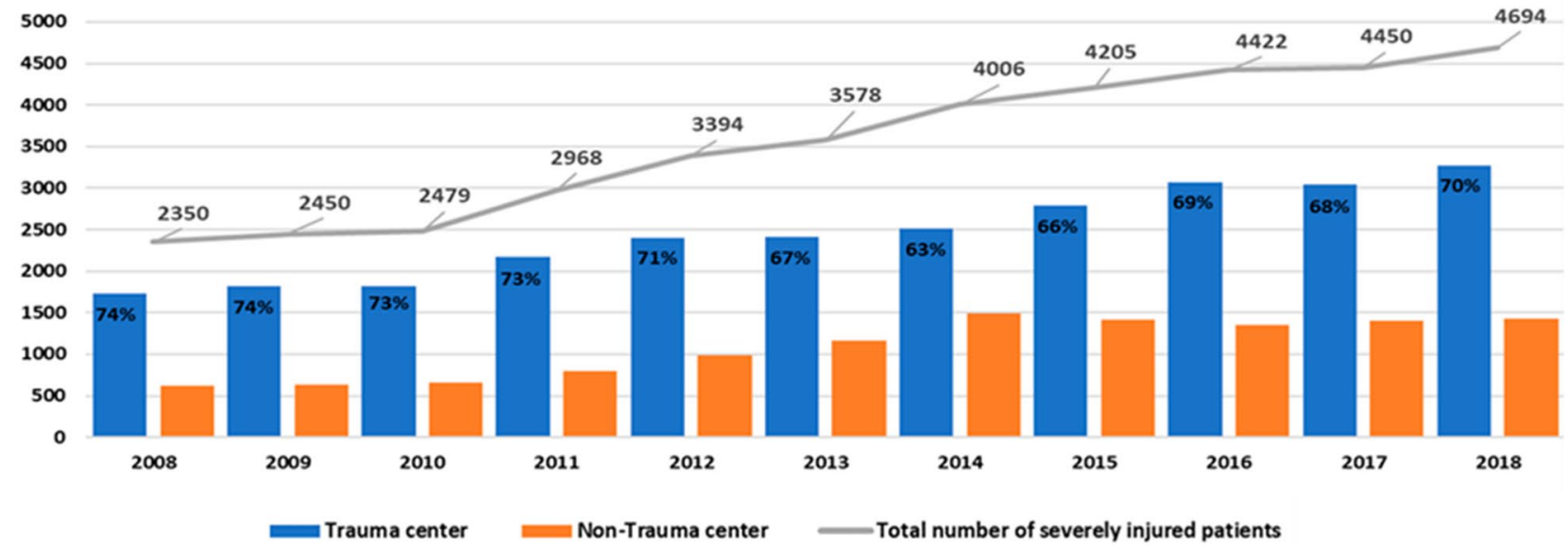

\section{B. Patients with severe neurotrauma AIS $\geq 3$}

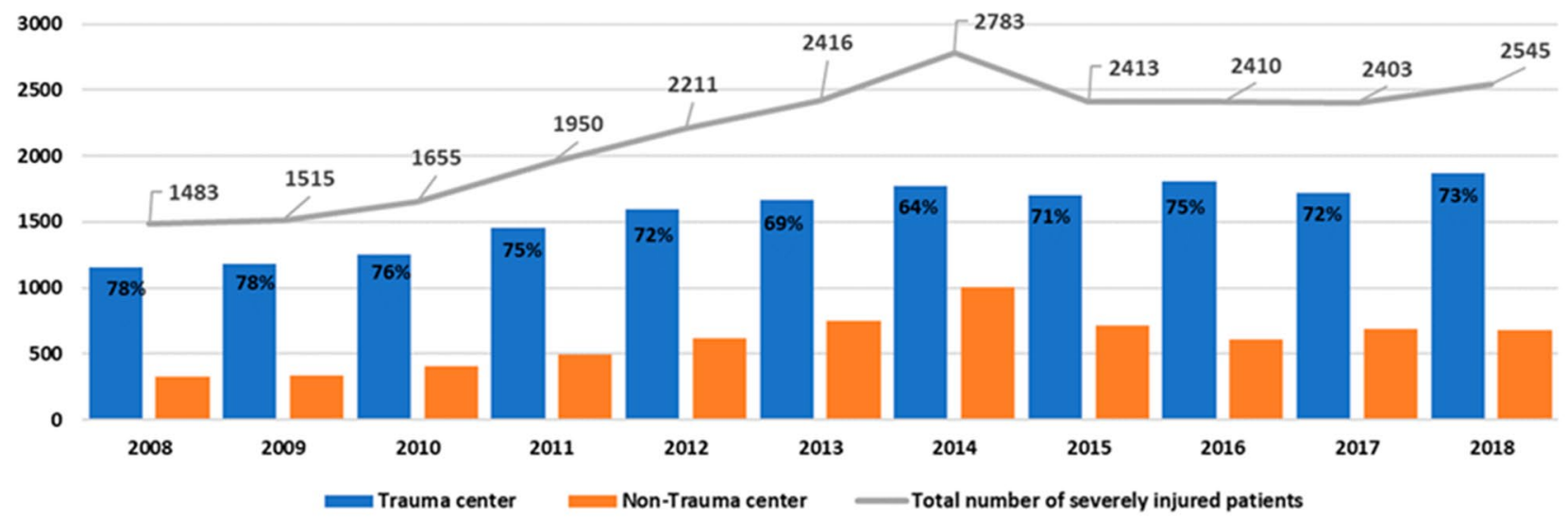

\section{Patients without severe neurotrauma}

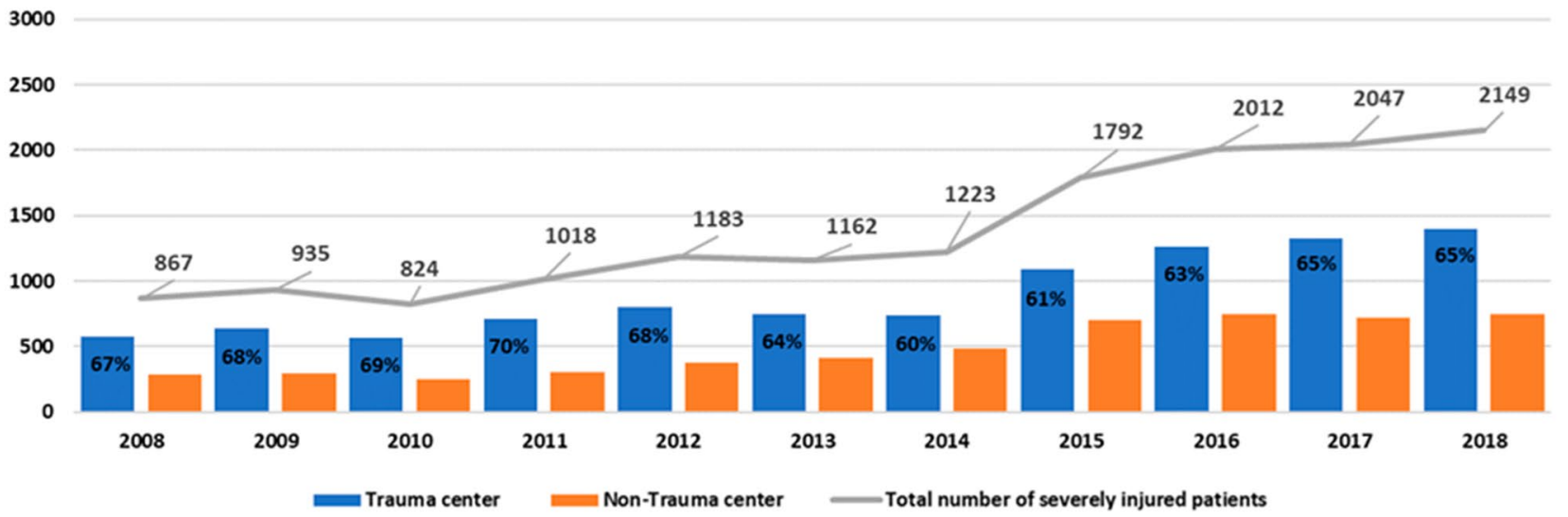

Fig. 2 Distribution of severely injured patients (ISS $\geq 16$ ) registered in the Dutch Trauma Registry, directly brought to a level I trauma center or to a non-trauma center over time for a all patients, and separately for patients $\mathbf{b}$ with and $\mathbf{c}$ without severe neurotrauma, by calendar year 


\section{Results}

\section{Distribution of severely injured patients}

The number of severely injured patients registered in the Dutch Trauma Registry increased from 2350 patients in 2008 to 4694 in 2018 [Fig. 2a]. At the same time, the number of participating NTCs varied from 76 to 100 (72-100\%; Table 1). In the years 2008-2014, on average, $66 \%$ of all documented severely injured patients had severe neurotrauma, while this was $55 \%$ over the years 2015-2018 (Table 1). Both the numbers of registered patients with severe neurotrauma (Fig. 2b) and without severe neurotrauma (Fig. 2c) increased over the 10-year period. The unadjusted proportion of all severely injured patients that were directly brought to a TC was $70 \%$ on average. This proportion decreased from 74 to $63 \%$ between 2008 and 2014, when the number of NTCs participating in the DTR still increased, and then increased to $70 \%$ in 2018 (Fig. 2a). A similar trend was seen in both the subgroup of patients with severe neurotrauma (Fig. 2b) and the subgroup of patients without neurotrauma or with only mild/moderate neurotrauma (Fig. 2c).

A similar trend in the proportion of severely injured patients that were directly brought to a TC was seen after adjustment for variation in case mix and for non-participation of NTCs (Fig. 3). After a decreasing trend between 2008 and 2014, the adjusted proportion increased over the years 2014-2016 and remained stable thereafter. On average, over the past decade, $73 \%$ of the patients with severe neurotrauma and $66 \%$ of patients without severe neurotrauma were directly transported to a TC.

\section{A. All patients}

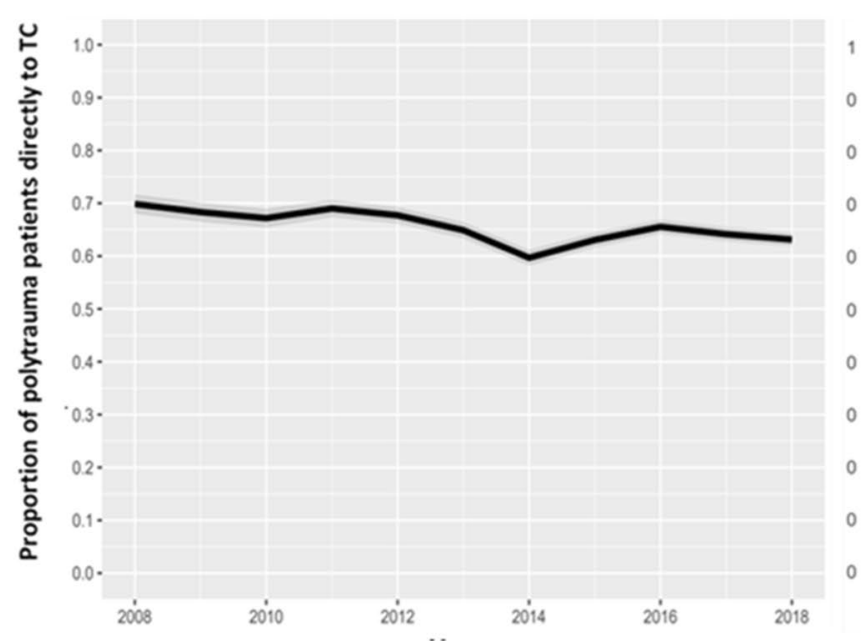

B. Patients with severe neurotrauma (AIS $\geq 3$ )

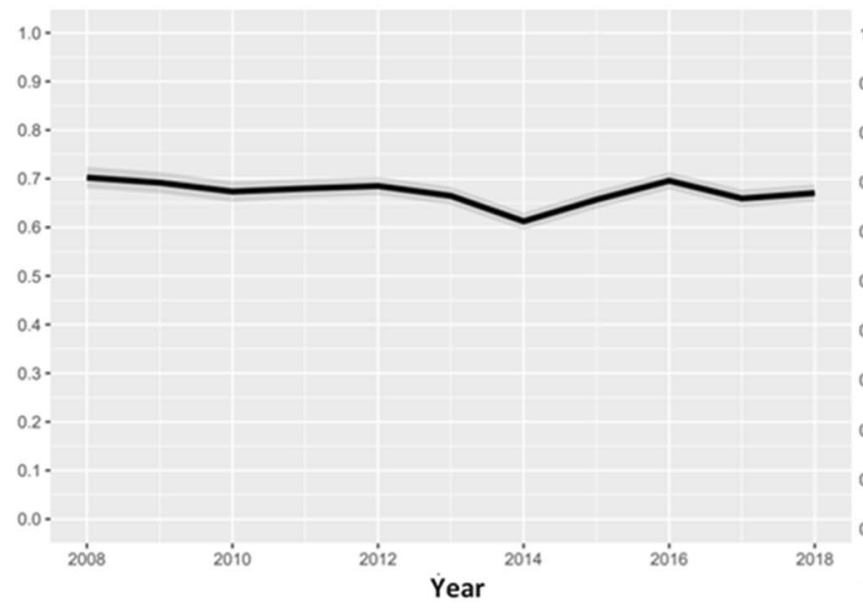

Fig. 3 Proportion of severely injured patients (ISS $\geq 16$ ) directly brought to a trauma center, after correction for difference in case mix and for non-participation in the Dutch Trauma Registry, for

\section{Patients without severe neurotrauma}

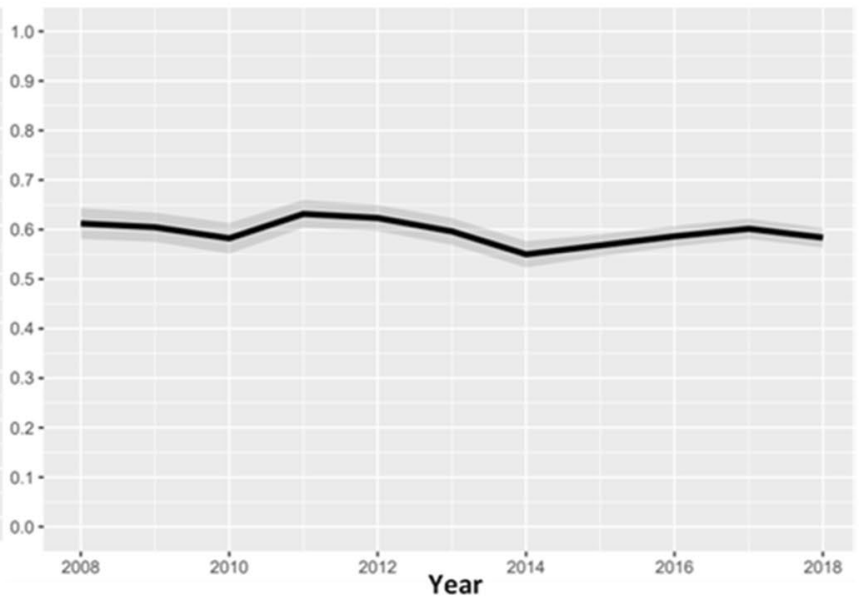

all patients (a), and separately for patients with (b) and without (c) severe neurotrauma, per calendar year 

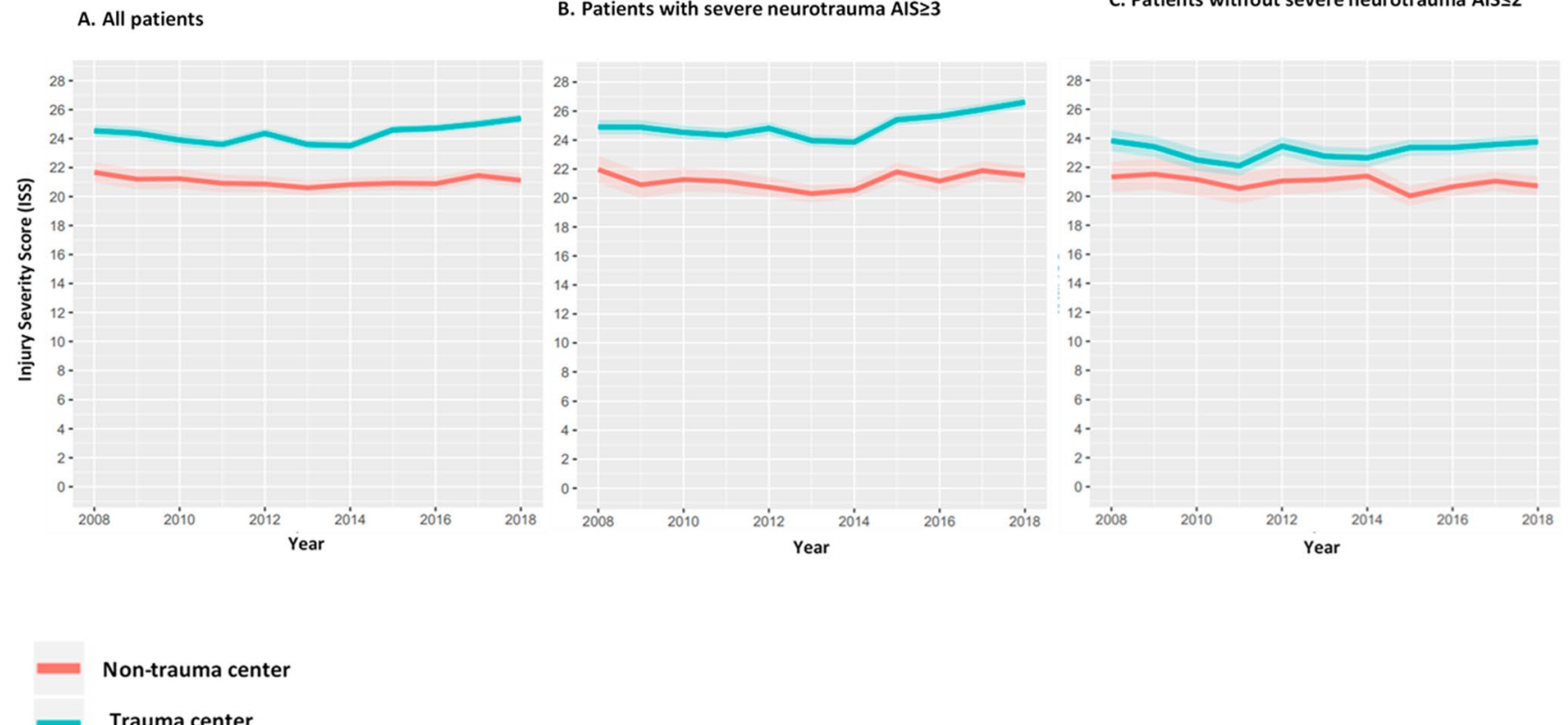

Fig. 4 Median Injury Severity Score (ISS) for severely injured patients (ISS $\geq 16$ ) directly brought to a trauma center or a non-trauma center, for a all patients, and separately for patients $\mathbf{b}$ with and (C) without severe neurotrauma, by calendar year
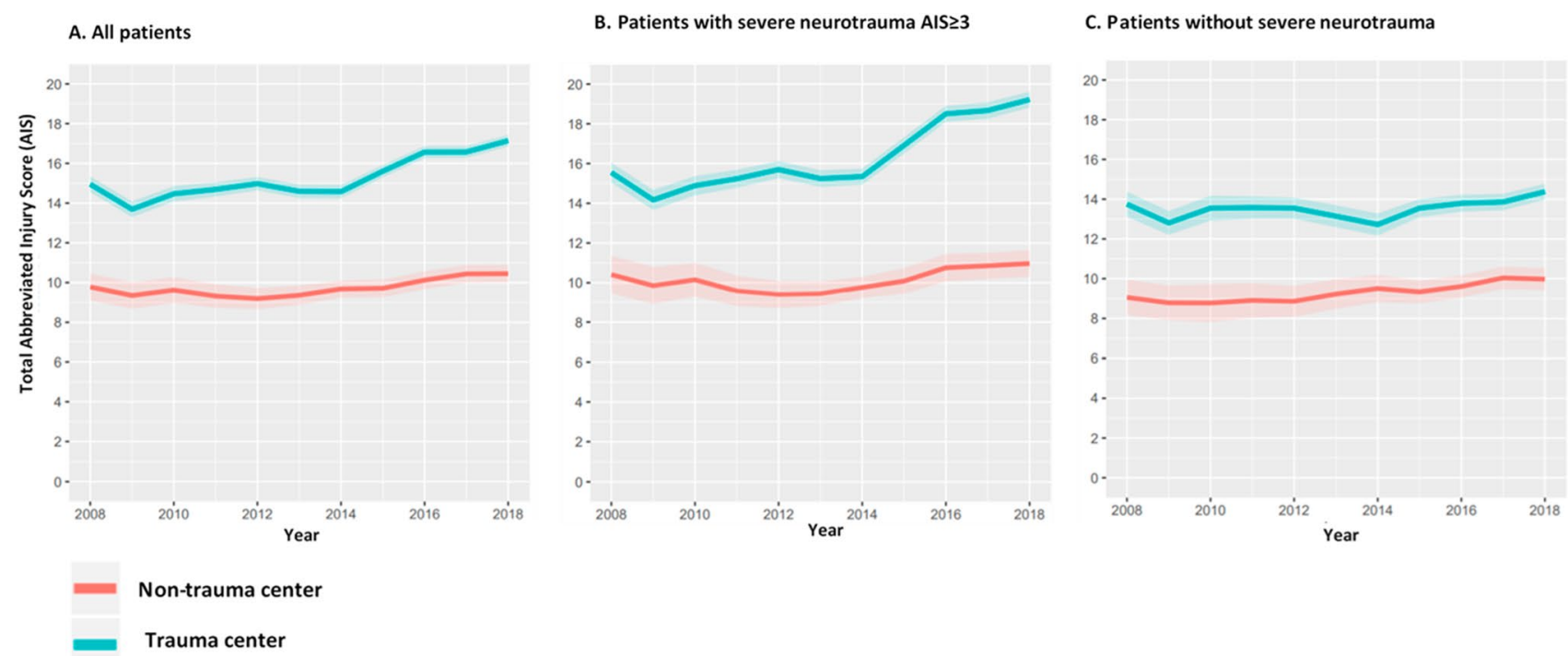

Fig. 5 Median total Abbreviated Injury Score (AIS) for severely injured patients (ISS $\geq 16$ ) directly brought to a trauma center or a non-trauma center, for $\mathbf{a}$ all patients, and separately for patients $\mathbf{b}$ with and $\mathbf{c}$ without severe neurotrauma, by calendar year

Similar trends were seen for patients with an ISS $>25$ (data not shown), with still $20 \%$ of the severely injured patients not primarily transported to a trauma center. Comparable to the ISS $\geq 16$ group, patients with severe head trauma (AIS $\geq 3$ ) had a higher chance of being primarily transported to a trauma center than patient without severe head trauma (AIS $<3$ ).

\section{Injury severity}

During the entire study period, the patients directly brought to a TC were more severely injured, reflected by a higher median ISS (22, interquartile range [IQR] 1727 ) and higher median total AIS (13, IQR 9-20), than the patients who were directly brought to a NTC (ISS 18, IQR 17- 25 and total AIS 
8, IQR 6-12) (Figs. 4 and 5). For all severely injured patients and for the subgroup of patients with severe neurotrauma, the total AIS and ISS in the patients directly brought to a TC (median ISS 24, IQR 17-29 and total AIS 14, IQR 9-21) increased from 2014 onwards (ISS 2014 22, IQR 17-27 vs ISS 2018 25, IQR 19-29 and total AIS 2014 13, IQR 9-20 vs total AIS 2018 17, IQR 12-24), while it remained steady over the years for the patients who were brought to a NTC (ISS 19, IQR 17-25 and total AIS 9, IQR 6-12) (Figs. 4a, $\mathrm{b}$ and $5 \mathrm{a}, \mathrm{b}$ ). For the subgroup of severely injured patients without severe neurotrauma, the median ISS and total AIS remained similar over time, for both the patients that were directly brought to a TC (ISS 20, IQR 17-25 and total AIS 12 , IQR 7-17) and for those directly brought to a NTC ( ISS 18, IQR 17-22 and total AIS 8, IQR 5-12) (Figs. 4c and 5c).

\section{Discussion}

Over the period 2008-2018, the centralization of trauma care in the Netherlands continued. The total number of registered severely injured patients has increased to annually about 4500. This increase is at least partly attributable to increased participation of NTCs in the DTR and to a more accurate registration. As of 2014, all TCs and NTCs with an ED participated in the registry and from then on representative data was available. The proportion of the severely injured patients who were directly brought to a TC slightly increased, and stabilized at $70 \%$ in the most recent years. This proportion was somewhat higher for the severely injured patients with severe neurotrauma than for those without neurotrauma or only mild or moderate neurotrauma. The injury severity within the group of severely injured patients that were directly brought to a TC has increased since 2014, especially in the subgroup of patients with severe neurotrauma.

Despite many improvements, challenges remain to be faced. As a consequence of the introduction of a trauma system, severely injured patients are more likely to be admitted to a TC than in the 1990s [6]. However, about 30\% of these patients are still transported to NTCs in the Netherlands. Similar percentages are seen in other countries, such as Norway and the United States [17-21]. According to the American College of Surgeons Committee on Trauma, an under-triage rate above $5 \%$ is unacceptable, as under-triage increases the risk of mortality and morbidity due to patients not being managed at the best-equipped hospital [22]. In addition, MacKenzie et al. showed in their study that, especially for the younger, more severely injured patients, treatment at a TC is not only more effective but also cost-effective, which underlines the importance of bringing the severely injured to a TC [23]. Studies show that especially the most severely injured patients, with ISS $\geq 25$, hemodynamically instable and patients with severe traumatic brain injury (AIS $\geq 3$ ), benefit the most from proper hospital triage, demonstrating lower mortality rates for these patients when brought to a TC [24-27]. Reducing under-triage should therefore be given priority. This does, however, remain a major challenge even in mature trauma systems [22, 28]. Van Rein et al. showed in their systematic review that almost all pre-hospital triage protocols had a low sensitivity and therefore failed to identify all severely injured patients who needed treatment in a TC [29]. Especially identifying serious neurotrauma by EMS providers has proven to be difficult; $32 \%$ of all neurotrauma and $21 \%$ of the severe neurotrauma are not recognized at the accident scene [30]. Particularly for these patients, the hospital triage may be further optimized by advanced triage tools. In trauma patients, the effects of drugs and alcohol often obscure the real trauma-related neurological symptoms so that symptoms often do not correspond with findings on the CT scans once the patients have arrived at the ED [30].

The current lack of field triage criteria able to adequately predict if a patient will be classified as severely injured contributes to the challenge to fulfill the Dutch Healthcare Institute's prerequisite of $90 \%$ severely injured patients being brought directly to a dedicated TC. In practice, emergency service providers guide their decision whether or not to go to a TC based on their clinical experience, and clinical signs of severe injury at the accident scene in addition to what the ambulance protocols prescribe [31]. Future research should focus on developing tools for scientifically substantiated assistance in this decision-making and improve the quality of pre-hospital triage in severely injured patients $[32,33]$.

\section{Strengths and limitations}

A strength of this study is that it includes data of all documented severely injured patients over a period of 10 years in one country. There are also some limitations that need to be addressed. We observed an increasing number of (severely) injured patients in the study period. Although we tried to correct for the fact that some NTCs did not participate at the beginning of the DTR, the increase in patient numbers may still, at least partly, be explained by the increasing NTC participation over the years. The increase in trauma patient numbers might also be caused by more accurate registration. Another potential bias was posed by the AIS conversion in 2015, when the way of injury coding in the DTR was changed from the 1998 version of the Abbreviated Injury Scale to the 2005/2008 update version. It is well known that the AIS08 version substantially differs from the AIS98 version with regard to the classification of injury severity and accuracy. Specifically, the AIS08 classification results in less patients being classified as severely injured patients (ISS $\geq 16$ ) and less patients with severe (AIS $\geq 3$ ) neurotrauma. This probably also explains the increase in numbers of patients with minor TBI and the reduction in numbers of 
severely injured patients with severe neurotrauma, which was on average $66 \%$ over the years 2008-2014 and 55\% over the years 2015-2018. This assumption is confirmed by Pal et al. who showed an increase in head AIS1 and AIS2 classifications and a decrease in AIS $>3$ or higher classifications after using the AIS2008 classification [34]. So, despite our best efforts in reclassifying the AIS98 to AIS08 codes according to Palmer's model [10], it remains challenging to combine the data of both classifications $[10,34,35]$.

Lastly, data on the pre-hospital assumption of injury severity are not available in the National Trauma Registry and could not be obtained from the emergency services due to the strict privacy regulations. Therefore, we could not combine the pre-hospital and National Trauma Registry data to give a better insight into the correlation between the triage and ISS.

\section{Conclusion}

Since the introduction of a well-organized trauma system in the Netherlands, trauma care has become progressively centralized, with more severely injured patients being brought directly to a TC. The injury severity within the group of severely injured patients that are directly transported to a TC has increased slightly in the most recent years, especially in the subgroup of patients with severe neurotrauma. However, still $30 \%$ of all severely injured patients is initially brought to a NTC. Future research should focus on improving prehospital triage to facilitate swift transfer of the right patient to the right hospital.

Author contributions All authors contributed to the conception and design of the research. SD contributed to the data collection and data analysis. All authors contributed to the data interpretation. SD drafted the manuscript. All authors critically revised the manuscript, read and approved the final manuscript, and agree to be fully accountable for ensuring the integrity and accuracy of the work.

Funding This research did not receive any specific grant from funding agencies in the public, commercial, or not-for-profit sectors.

Availability of data and material Data is available upon request.

\section{Compliance with ethical standards}

Conflicts of interest There are no conflicts of interest to report.

Open Access This article is licensed under a Creative Commons Attribution 4.0 International License, which permits use, sharing, adaptation, distribution and reproduction in any medium or format, as long as you give appropriate credit to the original author(s) and the source, provide a link to the Creative Commons licence, and indicate if changes were made. The images or other third party material in this article are included in the article's Creative Commons licence, unless indicated otherwise in a credit line to the material. If material is not included in the article's Creative Commons licence and your intended use is not permitted by statutory regulation or exceeds the permitted use, you will need to obtain permission directly from the copyright holder. To view a copy of this licence, visit http://creativecommons.org/licenses/by/4.0/.

\section{References}

1. ten Duis HJ, van der Werken C. Trauma care systems in The Netherlands. Injury. 2003;34(9):722-7.

2. Goris RJADJ. De zorg voor detrauma-patiënt: meer en beter onderzoek nodig. Medisch Contact. 1984;39:301-2.

3. Ministerie van Volksgezondheid Welzijn en Sport. Met Zorg Verbonden. 1997.

4. Nederlandse Vereniging voorTraumatologie. Traumazorg; onze zorg. 1997.

5. American College of Surgeons- Committee on Trauma. Resources for optimal care of the injured patients. American College of Surgeons,; Chicago, 19981999.

6. Twijnstra MJ, Moons KG, Simmermacher RK, Leenen LP. Regional trauma system reduces mortality and changes admission rates: a before and after study. Ann Surg. 2010;251(2):339-43. https://doi.org/10.1097/SLA.0b013e3181c0e910.

7. Baker SP, O'Neill B. THE INJURY SEVERITY SCORE: AN UPDATE. J Trauma Acute Care Surg. 1976;16(11):882-5.

8. Barrington I. The Abbreviated Injury Scale 1990 revision-update 98. Adv Automot Med. 1998.

9. Gennarelli TA, Wodzin E. The abbreviated injury scale 2005. Update. 2008;20082008.

10. Palmer CS, Franklyn M, Read-Allsopp C, McLellan S, Niggemeyer LE. Development and validation of a complementary map to enhance the existing 1998 to 2008 Abbreviated Injury Scale map. Scand J Trauma Resusc Emerg Med. 2011;19:29. https:// doi.org/10.1186/1757-7241-19-29.

11. Team RC. R development core team. RA Lang Environ Stat Comput. 2013.

12. Boyd CR, Tolson MA, Copes WS. Evaluating trauma care: the TRISS method. Trauma Score and the Injury Severity Score. J Trauma. 1987;27(4):370-8.

13. Sturms L. The Dutch Trauma Registry Facts and figures 2015. 2015. https://www.lnaz.nl/cms/files/symposium2016/6._Sturm s_2_nov_2016_LNAZ_symposium.pdf.

14. Ringdal KG, Skaga NO, Hestnes M, Steen PA, Roislien J, Rehn M, et al. Abbreviated Injury Scale: not a reliable basis for summation of injury severity in trauma facilities? Injury. 2013;44(5):691-9. https://doi.org/10.1016/j.injury.2012.06.032.

15. Maduz R, Kugelmeier P, Meili S, Doring R, Meier C, Wahl P. Major influence of interobserver reliability on polytrauma identification with the Injury Severity Score (ISS): Time for a centralised coding in trauma registries? Injury. 2017;48(4):885-9. https://doi. org/10.1016/j.injury.2017.02.015.

16. Mimasaka S, Ohtsu Y, Tsunenari S, Funayama M. Postmortem cytokine levels and severity of traumatic injuries. Int J Legal Med. 2006;120(5):265-70. https://doi.org/10.1007/s00414-006-0081-2.

17. Zorg LNA. Landelijke Traumaregistratie 2012-2016. Utrecht: Rapportage Nederland; 2017.

18. Jeppesen E, Cuevas-Østrem M, Gram-Knutsen C, Uleberg O. Undertriage in trauma: an ignored quality indicator? Scandinavian Journal of Trauma. Resuscitation Emerg Med. 2020;28(1):34. https://doi.org/10.1186/s13049-020-00729-6.

19. Uleberg O, Vinjevoll O, Eriksson U, Aadahl P, Skogvoll E. Overtriage in trauma-what are the causes? Acta Anaesthesiol Scand. 2007;51(9):1178-83. 
20. Newgard CD, Fu R, Zive D, Rea T, Malveau S, Daya M, et al. Prospective validation of the national field triage guidelines for identifying seriously injured persons. J Am Coll Surg. 2016;222(2):14658.e2. https://doi.org/10.1016/j.jamcollsurg.2015.10.016.

21. Xiang H, Wheeler KK, Groner JI, Shi J, Haley KJ. Undertriage of major trauma patients in the US emergency departments. Am J Emerg Med. 2014;32(9):997-1004. https://doi.org/10.1016/j. ajem.2014.05.038.

22. MacKenzie EJ, Rivara FP, Jurkovich GJ, Nathens AB, Frey KP, Egleston BL, et al. A national evaluation of the effect of traumacenter care on mortality. N Engl J Med. 2006;354(4):366-78. https://doi.org/10.1056/NEJMsa052049.

23. MacKenzie EJ, Weir S, Rivara FP, Jurkovich GJ, Nathens AB, Wang W, et al. The value of trauma center care. J Trauma. 2010;69(1):1-10. https://doi.org/10.1097/TA.0b013e3181e03a21.

24. Vickers BP, Shi J, Lu B, Wheeler KK, Peng J, Groner J et al. Comparative study of ED mortality risk of US trauma patients treated at level I and level II vs nontrauma centers. Am J Emerg Med. 2015;33(9):1158-65. doi: https://doi.org/10.1016/j. ajem.2015.05.010.

25. Harmsen AMK, Giannakopoulos GF, Moerbeek PR, Jansma EP, Bonjer HJ, Bloemers FW. The influence of prehospital time on trauma patients outcome: a systematic review. Injury. 2015;46(4):602-9. https://doi.org/10.1016/j.injury.2015.01.008.

26. Mills EHA, Aasbjerg K, Hansen SM, Ringgren KB, Dahl M, Rasmussen BS, et al. Prehospital time and mortality in patients requiring a highest priority emergency medical response: a Danish registry-based cohort study. BMJ Open. 2019;9(11):e023049. https://doi.org/10.1136/bmjopen-2018-023049.

27. Härtl R, Gerber LM, Iacono L, Ni Q, Lyons K, Ghajar J. Direct transport within an organized state trauma system reduces mortality in patients with severe traumatic brain injury. J Trauma Acute Care Surg. 2006;60(6):1250-6.

28. Newgard CD, Uribe-Leitz T, Haider AH. Undertriage remains a vexing problem for even the most highly developed trauma systems: the need for innovations in field triage. JAMA Surg. 2018;153(4):328.

29. van Rein EAJ, Houwert RM, Gunning AC, Lichtveld RA, Leenen LPH, van Heijl M. Accuracy of prehospital triage protocols in selecting severely injured patients: a systematic review. J Trauma Acute Care Surg. 2017;83(2):328-39. https://doi.org/10.1097/ ta.0000000000001516.

30. van Rein EAJ, Jochems D, Lokerman RD, van der Sluijs R, Houwert RM, Lichtveld RA, et al. Diagnostic value of emergency medical services provider judgement in the identification of head injuries among trauma patients. Eur J Neurol. 2019;26(2):274-80. https://doi.org/10.1111/ene.13804.

31. Newgard CD, Nelson MJ, Kampp M, Saha S, Zive D, Schmidt $\mathrm{T}$, et al. Out-of-hospital decision making and factors influencing the regional distribution of injured patients in a trauma system. J Trauma. 2011;70(6):1345-53. https://doi.org/10.1097/TA.0b013 e3182191a1b.

32. Voskens FJ, van Rein EAJ, van der Sluijs R, Houwert RM, Lichtveld RA, Verleisdonk EJ, et al. Accuracy of prehospital triage in selecting severely injured trauma patients. JAMA Surg. 2018;153(4):322-7. https://doi.org/10.1001/jamasurg.2017.4472.

33. van Rein EAJ, van der Sluijs R, Houwert RM, Gunning AC, Lichtveld RA, Leenen LPH, et al. Effectiveness of prehospital trauma triage systems in selecting severely injured patients: is comparative analysis possible? Am J Emerg Med. 2018. https:// doi.org/10.1016/j.ajem.2018.01.055.

34. Pal C, Hirayama S, Sangolla N, Manoharan J, Kulothungan V, Stewart W, et al. Effect of Abbrevieted Injury Scale (AIS) Change on Injury Severity Prediction for Advanced Automatic Crash Notification (AACN). Int J Autom Eng. 2017;8(2):71-8.

35. Palmer CS, Franklyn M. Assessment of the effects and limitations of the 1998 to 2008 Abbreviated Injury Scale map using a large population-based dataset. Scand J Trauma Resusc Emerg Med. 2011;19(1):1. https://doi.org/10.1186/1757-7241-19-1. 